\title{
Minimal Dirac Fermionic Dark Matter with Nonzero Magnetic Dipole Moment
}

\author{
Jae Ho Hed* \\ Physics Department, University of Illinois at Chicago, Chicago, Illinois 60607, USA
}

\begin{abstract}
A neutral Dirac fermion $\psi$ is supplied as a singlet within the context of the standard model (SM) and is considered as a dark matter $(\mathrm{DM})$ candidate near electroweak scale $(10 \sim 1000 \mathrm{GeV})$ with nonzero magnetic dipole moment. The Dirac particles have four different types of electromagnetic couplings (four form factors) in general. We expect that the candidate mainly interacts with SM particles through magnetic dipole moment (MDM), since MDM conserves the discrete symmetries like parity $(\mathrm{P})$, time reversal $(\mathrm{T})$, and charge conjugation $(\mathrm{C})$ or its combination $\mathrm{CP}$. The magnetic dipole moment constrained by the relic density may be as large as $10^{-18} \sim 10^{-17} e \mathrm{~cm}$. We show that the elastic scattering is due to a spin-spin interaction for the direct detection, and the predictions are under experimental exclusion limits of the current direct detectors, XENON10 and CDMS II. We also consider the possibility of WIMP detection in near future.
\end{abstract}

*Electronic address: jheo1@uic.edu 


\section{INTRODUCTION}

The identity of the dark matter (DM) has been one of the most important outstanding questions in physics and cosmology. It is known that DM must be nonbaryonic, nonrelativistic (cold) and nonluminous matter [1]. So it is not one of elementary particles contained in the standard moldel (SM). The leading candidate from particle theory is a weakly interacting massive particle (WIMP), since the annihilation cross section can lead to the correct thermal residual abundance with the right magnitude. The new stable massive particles could be generated in many extensions of the SM [2] [3] [4] [5] [6].

In this letter we consider a Dirac fermionic dark matter candidate with mass near electroweak scale $(10 \sim 1000 \mathrm{GeV})$, which is supplied as a singlet within the standard model context. The Dirac particles have four different types of electromagnetic couplings (four form factors) in general. Among the four couplings, we expect that magnetic dipole moment (MDM) is sizable for the neutral fermion. The electric charges must be prohibited by the unbroken $U(1)_{\mathrm{Q}}$ gauge symmetry [7] and the other couplings except for magnetic dipole accompany violations of the discrete symmetries. The violations of the discrete symmetries might be sizable for the DM, but the violations have been estimated to be small for the known Dirac particles. We assume that the candidate has the small violations of the discrete symmetries.

The singlet Dirac fermion $\psi$ should couple to the shifted photon (hypercharge gauge boson $B)$ near electroweak scale $(10 \sim 1000 \mathrm{GeV})$, instead of photon. The Lagrangian with the magnetic dipole moment of $B, \mu_{B}$, is

$$
\mathcal{L}_{e f f}=\frac{1}{2} \mu_{B} \bar{\psi} \sigma_{\mu \nu} \psi B^{\mu \nu}
$$

In the standard model context, the Lagrangian may be expressed about photon and $Z$ boson.

$$
\mathcal{L}_{e f f}=\frac{1}{2} \mu \bar{\psi} \sigma_{\mu \nu} \psi\left(F^{\mu \nu}-\tan \theta_{W} Z^{\mu \nu}\right)
$$

where $F^{\mu \nu}$ is the field strength for photon, $Z^{\mu \nu}$ for $Z$ boson and $\mu$ is the magnetic dipole moment ${ }^{1}$.

\footnotetext{
${ }^{1}$ To be strict, this is a magnetic dipole form factor, $F_{2}\left(q^{2}\right)$. The magnetic dipole moment is defined at
} 


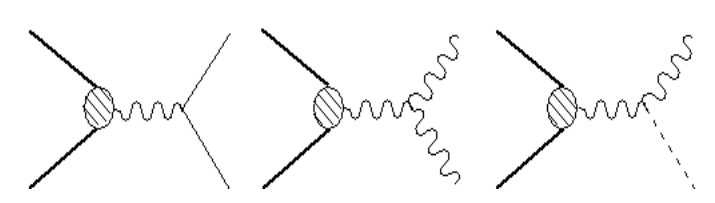

FIG. 1: Feynman diagrams for WIMP pair annihilation into $f \bar{f}, W W, Z H$. The hatched circle indicates the vertex for the dipole coupling.

\section{ANNIHILATION PROCESS}

In the leading order of the magnetic dipole moment, WIMP annihilates into SM particles $(f \bar{f}, W W, Z H)$ via the $s$-channel $\gamma, Z$ exchanges, where the Feynman diagrams are given in Fig.1. The MDM operator is split into energy and momentum dependent parts by the familiar Gordon identity.

$$
\bar{v}\left(p^{\prime}\right) \sigma^{\mu \nu} q_{\nu} u(p)=i \bar{v}\left(p^{\prime}\right)\left(2 M \gamma^{\mu}+\left(p^{\prime}-p\right)^{\mu}\right) u(p)
$$

The MDM operator has energy dependence. This implies that there is no threshold or helicity suppression due to violations of discrete symmetries. There is also no lepton number violation for this annihilation process, since the candidate is a Dirac particle. The below calculation for annihilation rates confirm this fact.

The squared scattering amplitude in an average over spin of initial states and a sum over colors and spins of final states is

$$
|\mathcal{M}|^{2}=\frac{N_{C}}{4} \mu^{2}\left|Q_{\gamma} P_{\gamma}-Q_{Z} \tan \theta_{W} P_{Z}\right|^{2} L^{\mu \nu} Q^{\rho \sigma} g_{\mu \rho} g_{\nu \sigma}
$$

where $Q_{\gamma}, Q_{Z}$ are couplings involved the outgoing particles with photon and $Z$ boson, $P_{\gamma} \equiv$ $1 / s, P_{Z} \equiv 1 /\left[\left(s-m_{Z}^{2}\right)+i m_{Z} \Gamma_{Z}\right]$ imply photon and $Z$ boson propagations, and $N_{C}$ is number of colors for the final states, 3 for quarks and 1 for the other particles. $L^{\mu \nu}$ is the trace of initial states and $Q^{\rho \sigma}$ stands for the trace of final states.

\footnotetext{
$q^{2}=0$. However we do not distinguish the difference of both terminologies in this letter.
} 


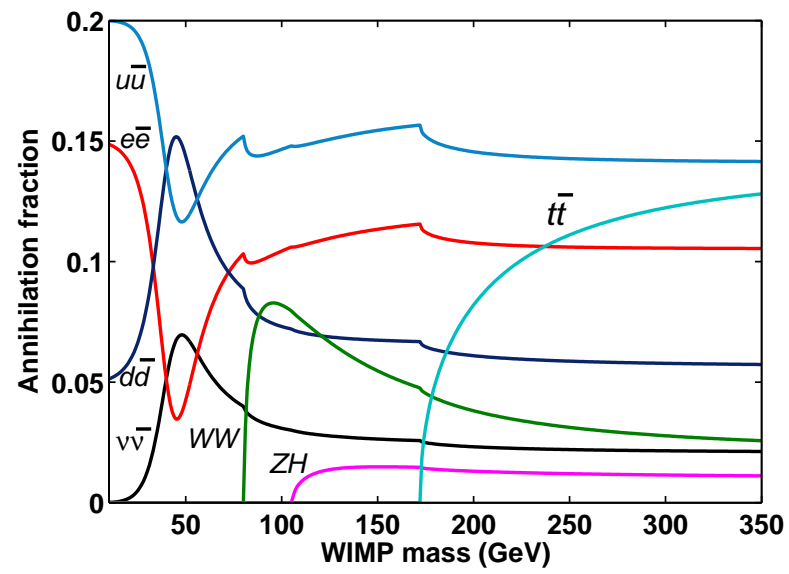

FIG. 2: (color online) Annihilation fraction of particles. The fermionic annihilation products dominate, hadronic annihilation products in particular.

The consistant annihilation rates for each final state are

$\sigma_{f} v_{\text {rel }}=\sum_{f} \frac{N_{C} \alpha \beta_{f}}{3}\left[\left|e_{f} P_{\gamma}-\frac{c_{V} P_{Z}}{2 \cos ^{2} \theta_{W}}\right|^{2}\left(s+2 m_{f}^{2}\right)+\left|\frac{c_{A} P_{Z}}{2 \cos ^{2} \theta_{W}}\right|^{2}\left(s-4 m_{f}^{2}\right)\right] \cdot \mu^{2}\left(s+8 M^{2}\right)$

$\sigma_{W} v_{\text {rel }}=\frac{\alpha \beta_{W} s}{24}\left|P_{\gamma}-P_{Z}\right|^{2} \frac{s^{2}+20 s m_{W}^{2}+12 m_{W}^{4}}{m_{W}^{4}} \cdot \mu^{2}\left(s+8 M^{2}\right)$

$\sigma_{Z H} v_{\text {rel }}=\frac{\alpha|\boldsymbol{p}|}{2 \sqrt{s}}\left|\frac{m_{Z}^{2}}{m_{W} \cos ^{2} \theta_{W}} P_{Z}\right|^{2}\left(1+\frac{\boldsymbol{p}^{2}}{3 m_{Z}^{2}}\right) \cdot \mu^{2}\left(s+8 M^{2}\right)$

where $v_{\text {rel }}$ is the relative velocity of the annihilating particles, $\alpha$ is the electric fine structure constant, $\beta$ is the velocity of the particle, $c_{V}=T_{f}^{3}-2 e_{f} \sin ^{2} \theta_{W}, c_{A}=T_{f}^{3}$ are the vector and axial couplings of $Z$-boson with electric charge $e_{f}$ and the third component of isospin $T_{f}^{3}$, and $|\boldsymbol{p}|=\frac{1}{2 \sqrt{s}}\left(\left(s-m_{H}^{2}+m_{Z}^{2}\right)^{2}-4 s m_{Z}^{2}\right)^{1 / 2}$ is momentum of $Z$-boson or Higgs. The total annihilation rate is $\sigma v_{\text {rel }}=\left(\sigma_{f}+\sigma_{W}+\sigma_{Z H}\right) v_{\text {rel }}$. We show the explicit mass dependence of fermions.

Fig.2 shows the annihilation fraction as a function of WIMP mass for the final states. The fermionic annihilation products dominate, particularly hadronic annihilation for entire parameter space. $Z H$ channel has the small contribution since it interacts with WIMP via only $Z$-boson. The $\gamma-Z$ interference plays very important role for the $W$-boson channel ${ }^{2}$,

\footnotetext{
${ }^{2}$ If we only consider the interaction with photon, the contribution by $W$-boson becomes extremely large in high energies. The interaction with $Z$-boson avoids this danger.
} 
since the $W$-boson channel contribution results in a significant decrease in high energy due to the interference.

\section{CONSTRAINT FROM RELIC ABUNDANCE}

In the very early universe, these particles would be present in large numbers in thermal equilibrium. They could reduce their density only through pair annihilation as universe is cool. This pair annihilation would be efficient enough to reduce the present day number density of WIMP. The time evolution of the relic density is described by the Boltzmann equation and we can solve the equation in the approxmate way [8]. After the freeze out, the actual number of the dark matter per comoving volume becomes constant and the present relic density is determined. The relic density is roughly

$$
\Omega_{\mathrm{CDM}} h^{2} \simeq \frac{\left(1.07 \times 10^{9}\right) x_{F}}{\sqrt{g_{*}} m_{P l}(\mathrm{GeV})\left\langle\sigma v_{\mathrm{rel}}\right\rangle}
$$

where $g_{*}$ is the effective degrees of freedom in equilibrium, $m_{P l}$ is Planck mass, $x_{F}$ is the inverse freeze out temperature and $\left\langle\sigma v_{\text {rel }}\right\rangle$ is the thermal average of annihilation rate.

The inverse freeze out temperature has almost no dependence on WIMP mass, $x_{F} \simeq 20$ near electroweak scale. The present relic density of CDM measured from WMAP (Wilkinson microwave anisotropy probe) data [9] is $\Omega_{\mathrm{CDM}} h^{2}=0.111 \pm 0.012$ at the level of $10 \%$ accuracy. So the thermal average of annihilation rate is $\left\langle\sigma v_{\text {rel }}\right\rangle \simeq 0.62 \mathrm{pb}$.

We take the thermal average for the annihilation obtained with Maxwell-Botzmann statistics, which is described in Ref. [10].

$$
\left\langle\sigma v_{\text {rel }}\right\rangle=\frac{x}{8 M^{5} K_{2}^{2}(x)} \int_{4 M^{2}}^{\infty} d s \sigma(s)\left(s-4 M^{2}\right) \sqrt{s} K_{1}\left(\frac{\sqrt{s}}{M} x\right)
$$

where $K_{i}$ is a modified Bessel function of order $i$.

Combination of Eq. (5) and (7) gives the allowed parameter sets for the magnetic dipole moment of WIMP. Fig.3 shows the allowed parameter sets which satisfy the WMAP measurements for relic density, in the case that the Dirac fermions account for all the observed dark matter. So those become the upper limits of WIMP magnetic dipole moment. All the annihilation rates of Eq.(5) are included and the known inputs are used as well as Higgs mass of $120 \mathrm{GeV}$. The anomaly indicates the resonant region of the $Z$ boson exchange where 


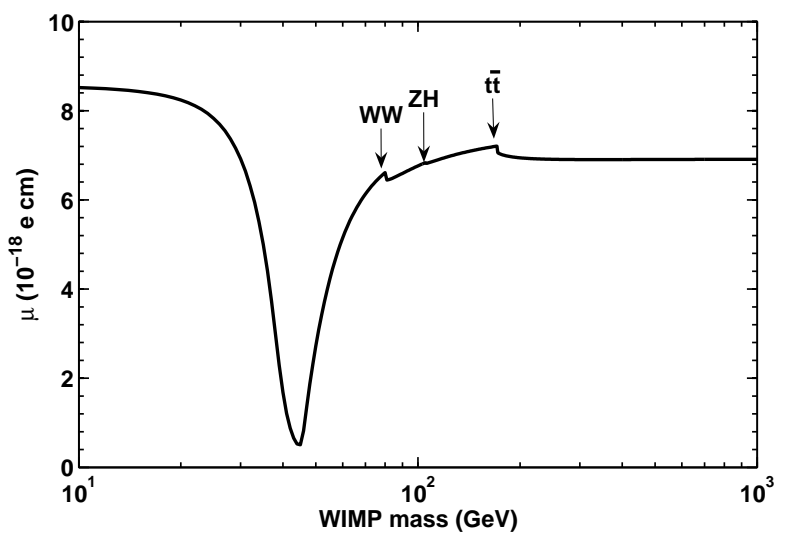

FIG. 3: Allowed parameter sets from the relic abundance. The abundance is chosen to be $\Omega_{\mathrm{CDM}} h^{2}=0.111$. The arrows indicate the points where new channels start.

$2 M \simeq m_{Z}$. In this region, the dipoles are relatively small in order to compensate the enhancement of annihilation rate by $Z$ boson resonace effect. The WIMP magnetic dipole mmoment may be as large as $10^{-18} \sim 10^{-17} e \mathrm{~cm}$. If the dipole comes from new physics effect, we predict the new physics with $O(\Lambda) \sim 10 \mathrm{TeV}$.

\section{DIRECT DETECTION}

The sensitivity of dark matter is controlled by their elastic scattering with a nucleus in a detector. The Feynman diagram which give rise to the WIMP-nucleon interaction is shown in Fig.4, which the $t$-channel exchanges of $\gamma, Z$ are only possible. For the cold dark matter, the momentum transfer is quite low, $|\boldsymbol{q}| \sim O(\mathrm{MeV})\left(E_{R} \sim O(\mathrm{KeV})\right)$. The interaction via $Z$ boson is $1 / m_{Z}^{2} \sim G_{F}$ where $G_{F}$ is the Fermi constant, and $1 / \boldsymbol{q}^{2}$ for the photon. The interaction via $Z$ boson may be neglected. We thus only consider the interaction via photon.

\section{A. Elastic-scattering cross section}

The effective Lagrangian may be described by four fermion interaction,

$$
\mathcal{L}_{q}=i \frac{\mu e e_{q}}{\boldsymbol{q}^{2}}\left(\bar{\psi} \sigma^{\mu \nu} q_{\nu} \psi\right)\left(\bar{q} \gamma_{\mu} q\right)
$$

where $e$ is the electric coupling and $e_{q}$ is an electric charge for the quark $q$. 


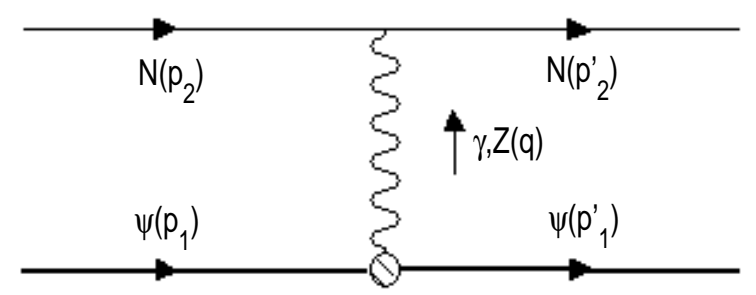

FIG. 4: Feynman diagram relevant to WIMP-nucleon elastic scattering. The hatched circle indicates the vertex for the dipole coupling.

The MDM operator $\bar{\psi} \sigma^{\mu \nu} q_{\nu} \psi$ has no energy dependence for the elastic scattering. So we take only space component of the operator and the leading order of $|\boldsymbol{q}|$ in the nonrelativistic expansion of the spinor. It results in the spin-spin insteraction between WIMP and quark.

The effective Lagrangian for nucleons may be expressed by the spin distrbution of quarks.

$$
\mathcal{L}_{N} \supset 2 i M \frac{\mu e a_{N}}{\boldsymbol{q}^{2}}\left(\bar{\psi} \gamma^{i} \psi\right)\left(\bar{N} \gamma_{i} N\right)
$$

where the symbol $N$ stands for proton $(p)$ or neutron $(n)$, and $a_{N} \equiv \sum e_{q} \Delta^{(N)} q$ where $\Delta^{(N)} q$ is the fraction of nucleon spin carried by quarks of type $q$. The fractions for proton, according to Ref.[11], are $\Delta^{(p)} u=0.78, \Delta^{(p)} d=-0.48$, and $\Delta^{(p)} s=-0.15$, each with an uncertainty of about \pm 0.02 , and $\Delta^{(n)} u=\Delta^{(p)} d, \Delta^{(n)} d=\Delta^{(p)} u, \Delta^{(n)} s=\Delta^{(p)} s$ for neutron.

The differential cross section of WIMP-nucleus in a spin average of initial states and a spin sum of final states is given by

$$
\frac{d \sigma_{e l}}{d \boldsymbol{q}^{2}}=\frac{\mu^{2} a_{N}^{2}}{4 \pi v^{2} \boldsymbol{q}^{4}}\left(\frac{e}{2 m}\right)^{2} \frac{1}{(2 S+1)(2 J+1)} \sum_{j, j^{\prime}, s, s^{\prime}}|\mathcal{M}|^{2}
$$

where $S=\frac{1}{2}$ is the WIMP spin, $J$ is the nuclear spin and $m$ is mass of the atomic nucleus.

The squared scattering amplitude can split into two parts, WIMP and nuclear parts. The WIMP part is

$$
\frac{1}{(2 S+1)} \sum_{s, s^{\prime}}|\mathcal{M}|_{\text {WIMP }}^{2, i j}=4 \frac{S(S+1)}{3}\left(\boldsymbol{q}^{2} \delta^{i j}-q^{i} q^{j}\right)
$$

The squared amplitude for the nuclear part depends on the nuclear structure function, $S(|\boldsymbol{q}|)$, which is normalized by the nuclear spin. 


$$
\frac{1}{(2 J+1)} \sum_{j, j^{\prime}}|\mathcal{M}|_{\text {nucleus }}^{2, i j}=\frac{J(J+1)}{3} S(|\boldsymbol{q}|)\left(\boldsymbol{q}^{2} \delta^{i j}-q^{i} q^{j}\right)
$$

The nuclear structure function $S(|\boldsymbol{q}|)$ may be expressed in terms of three form factors, isoscalar $\left(a_{0}\right)$, isovector $\left(a_{1}\right)$ and the inteference $\left(a_{0} a_{1}\right)$.

$$
S(|\boldsymbol{q}|)=a_{0}^{2} S_{00}(|\boldsymbol{q}|)+a_{0} a_{1} S_{01}(|\boldsymbol{q}|)+a_{1}^{2} S_{11}(|\boldsymbol{q}|)
$$

with $a_{0}=a_{p}+a_{n}$ and $a_{1}=a_{p}-a_{n}$. The functions $S_{i j}$ encompass the magnitude of the spin associated with the nucleon populations, as well as the effects of the spatial distribution of that spin at nonzero momentum transfers.

The WIMP-nucleus differential cross section results in

$$
\frac{d \sigma_{e l}}{d \boldsymbol{q}^{2}}=\frac{\mu^{2} \mu_{A}^{2}}{6 \pi v^{2}} \frac{J+1}{J} F^{2}(|\boldsymbol{q}|)
$$

where $\mu_{A}^{2} \equiv\left(\frac{e}{2 m} J\right)^{2} S(0)$ is the magnetic dipole moment of the atomic nucleus and $F^{2}(|\boldsymbol{q}|) \equiv$ $S\left(\left|\boldsymbol{q}^{2}\right|\right) / S(0)$ is the normalized form factor, $F^{2}(0)=1$. We have to integrate over $\boldsymbol{q}^{2}$ for the actual cross section. There are two quantities which depend on atomic nuclei, nuclear spin $J$ and the nuclear structure function $S\left(\left|\boldsymbol{q}^{2}\right|\right) \sim \mu_{A}^{2} F^{2}(|\boldsymbol{q}|)$. Those become the reasons we have the different signatures for the various targets.

The cross section at $|\boldsymbol{q}|=0$ is

$$
\sigma=\int_{0}^{4 M_{r}^{2} v^{2}} \frac{d \sigma_{e l}(|\boldsymbol{q}|=0)}{d \boldsymbol{q}^{2}} d \boldsymbol{q}^{2}=\frac{2}{3 \pi} M_{r}^{2} \mu^{2} \mu_{A}^{2} \frac{J+1}{J}
$$

where $M_{r}=M m /(M+m)$ is the reduced WIMP-nucleus mass.

The WIMP-nucleon cross sections result in

$$
\sigma_{p, n}=\frac{1}{2 \pi} m_{r}^{2} \mu^{2}\left(\frac{e a_{p, n}}{2 m}\right)^{2} \frac{J+1}{J}
$$

where $m_{r}=M m_{N} /\left(M+m_{N}\right)$ is the reduced WIMP-nucleon mass. This cross section still includes one of the target properties, nuclear spin $J$, so it is not totally the nucleus independent quantity. 


\section{B. WIMP detection}

The expected event rate depends on WIMP-nucleus cross section and the WIMP flux on the Earth, so the differential event rate per unit target mass and unit time is

$$
\frac{d R}{d \boldsymbol{q}^{2}}=\frac{\rho_{D}}{m M} \int v f(v) \frac{d \sigma_{e l}}{d \boldsymbol{q}^{2}} d v
$$

where $\rho_{D} \simeq 0.3 \mathrm{GeV} / \mathrm{cm}^{3}$ is the local DM density in the solar vicinity and $f(v)$ is the distribution of speeds relative to the dectector. We take the Maxwellian distribution considered the annual modulation [12].

$$
f(v) d v=\frac{v}{v_{E} v_{0} \sqrt{\pi}}\left\{\exp \left[-\frac{\left(v-v_{E}\right)^{2}}{v_{0}^{2}}\right]-\exp \left[-\frac{\left(v+v_{E}\right)^{2}}{v_{0}^{2}}\right]\right\} d v
$$

where $v_{0}=220 \mathrm{~km} / \mathrm{s}$ is the circular speed of the Sun around the Galactic center and $v_{E} \sim v_{0}$ is the Earth speed to the Sun. $v_{E}$ changes as the Earth's motion come into and out of alignment with the Sun's motion around the Galaxy. This causes an yearly modulation in the event rate which peaks around June 2nd each year [13]. We take the average velocity, $v_{E}=232 \mathrm{~km} / \mathrm{s}$.

With the relation $\boldsymbol{q}^{2}=2 m E_{R}$, Eq. (13), (16) and (17) lead to the differential event rate.

$$
\frac{d R}{d E_{R}}=\frac{\rho_{D} \mu^{2} \mu_{A}^{2}}{3 \pi M} \frac{(J+1)}{J} F^{2}\left(E_{R}\right) \int_{v_{\min }}^{\infty} \frac{f(v)}{v} d v
$$

The final formula for the event rate per unit target mass and unit time is given by

$$
R=\frac{\rho_{D} \sigma}{2 M M_{r}^{2}} \int_{E_{R, \text { min }}}^{E_{R, \max }} d E_{R} F^{2}\left(E_{R}\right) \cdot \frac{1}{2 v_{E}}\left[\operatorname{erf}\left(\frac{v_{\min }+v_{E}}{v_{0}}\right)-\operatorname{erf}\left(\frac{v_{\min }-v_{E}}{v_{0}}\right)\right]
$$

where $v_{\min }=\sqrt{\frac{E_{R, \min m} m}{2 M_{r}^{2}}}$. The form factor has to be integrated over $E_{R}$ in the recoil energy range. The form factors for the spin-spin interaction are relatively slowly decreased, so it results in suppressions for the event rates.

There are various isotope targets which have been considered in WIMP detection. Among them, we consider the isotope targets, ${ }^{73} \mathrm{Ge}$ and ${ }^{131} \mathrm{Xe}$, which have been used at the most sensitive detectors, CDMS II and XENON10. The recent estimated experimental sensitivities are 0.6 event $/ \mathrm{kg} /$ day for XENON10 [14] and CDMS II [15], and the planned experimental 
TABLE I: Current and planned Dark Matter detectors. XENON10 and CDMS II are the most sensitive detectors and SuperCDMS is a future detector.

\begin{tabular}{ccccc}
\hline \hline Experiment & Recoil energy range & Target & Spin & Mass $(\mathrm{Kg})$ \\
\hline XENON10 & $4.5 \sim 27 \mathrm{KeV}$ & ${ }^{131} \mathrm{Xe}$ & $\frac{3}{2}$ & $5.4 \mathrm{Kg}$ \\
CDMS II & $10 \sim 100 \mathrm{KeV}$ & ${ }^{73} \mathrm{Ge}$ & $\frac{9}{2}$ & $100 \mathrm{Kg}$ \\
SuperCDMS & $15 \sim 45 \mathrm{KeV}$ & ${ }^{73} \mathrm{Ge}$ & $\frac{9}{2}$ & $100 \mathrm{Kg}$ \\
\hline \hline
\end{tabular}

sensitivity is $1 \times 10^{-4}$ event $/ \mathrm{kg} /$ day at SuperCDMS [16]. The recoil energy ranges are listed at Table II for the corresponding detectors.

Fig.5 shows the WIMP-nucleon cross sections from Eq.(15) with the experimental limits in the WIMP mass range $10 \sim 1000 \mathrm{GeV}$. The predicted curves and the experimental limit contours are for pure proton coupling $\left(a_{n}=0\right)$ and pure neutron coupling $\left(a_{p}=0\right)$. The predicted curves show a little different from both isotope targets because of the nuclear spin effect. The form factors related to the spin structure functions are followed by Dimitrov et al. [17] for ${ }^{73} \mathrm{Ge}$ nucleus and Ressell et al. [18] for ${ }^{131}$ Xe nucleus, which are consistent with the experimental measurements for MDM in the reasonable precision. The ${ }^{73} \mathrm{Ge}$ and ${ }^{131} \mathrm{Xe}$ nuclei both contain an unpaired neutron, so the sensitivity to pure-neutron coupling is stronger. The predictions are under the current discovery limits for the entire mass range and are relatively close to the experimental limits. We should not discard the WIMP discovery through the magnetic dipole moment in near future.

\section{CONCLUSION AND DISCUSSION}

A Dirac fermion which was supplied as a singlet within the standard model context may mainly interact with the ordinary matters through magnetic dipole moment (MDM). It must be more general that the candidate couples to the shifted photon (hypercharge gauge boson) than coupling to photon directly near electroweak scale $10 \sim 1000 \mathrm{GeV}$. Assuming that the Dirac fermions account for all the observed dark matter, the MDM constrained by the relic density may be as large as $10^{-18} \sim 10^{-17} \mathrm{e} \mathrm{cm}$. The WIMP with nonzero MDM interacts with targets through MDM of the target nuclei and the cross sections are under the current experimental limits. Since the preditions are close to the current experimental limits, the 

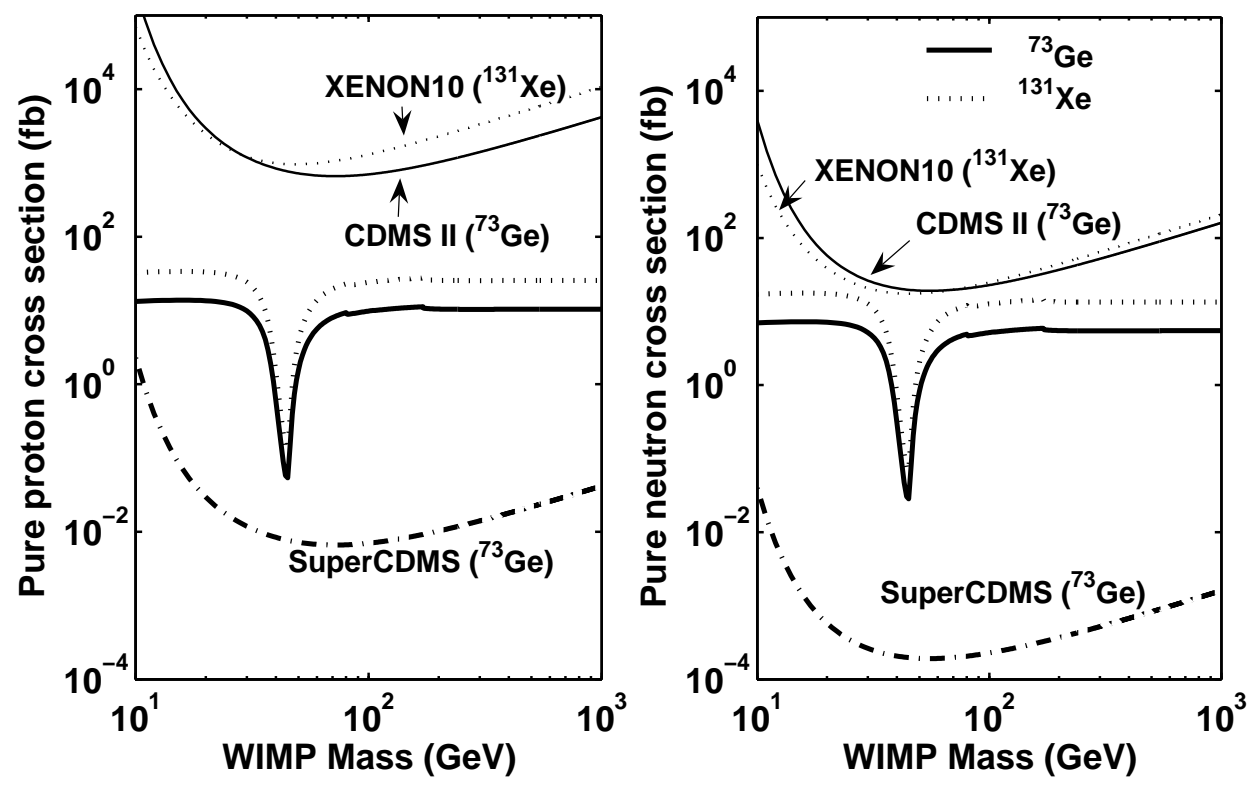

FIG. 5: The predictions for WIMP-nucleon cross sections, $\sigma_{p, n}$, as a function of WIMP mass. The light dotted lines are the exclusion limits from the XENON10 experiment and the solid lines from CDMS II experiment. Also shown by dash-dotted lines are the experimental sensitivities of the planned detector SuperCDMS, respectively.

detection of the WIMP through MDM should be considered be in near future.

Besides the direct search of WIMP, there are several experiments to search for dark matter indirectly, such like neutrino telescopes, $\gamma$-ray telescopes and antimatter telescopes. They might give the more serious constraints [19], but the indirect searches for dark matter accompany uncertain informations in astrophysics. This scenario, which the dark matter has Dirac fermionic nature and mainly interacts with the ordinary matters through the magnetic dipole moment, might not be ruled out.

We have also studied the possibility of WIMP detection with the method of a photon or jet tagging in ILC and LHC. Several tens or hundreds of WIMP events are expected, but these signals suffer the huge irreducible background come from the neutrino channel. So the 
expected signals are swamped by the irreducible background.

[1] A. Lange et al., Phys. Rev. D 63, 042001 (2001) arXiv:astro-ph/0005004] ; N.W. Halverson et al., Astrophys. J. Suppl. 148, 175 (2003) arXiv:astro-ph/0302209

[2] G. Jungman, M. Kamionkowski and K. Griest, Phys. Rep. 267, 195 (1996) ; K. Griest and M. Kamionkowski, Phys. Rept. 333, 167 (2000)

[3] A. Birkedal, A. Noble, M. Perelstein and A. Spray, Phys. Rev. D 74, 035002 (2006) arXiv:hep-ph/0603077

[4] M. Kakizaki, S. Matsumoto, Y. Sato and M. Senami, Phys. Rev. D 71, 123522 (2005) arXiv:hep-ph/0508283

[5] C.P. Burgess, M. Pospelov and T. Veldhuis, Nucl. Phys. B619, 709 (2001) arXiv:hep-ph/0011335; $\quad$ J. McDonald, Phys. Rev. D 50, 3637-3649 (1994) arXiv:hep-ph/0702143

[6] Y.G. Kim, and K.Y. Lee, Phys. Rev. D 75, 115012 (2007) arXiv:hep-ph/0611069 ; Y.G. Kim, K.Y. Lee and S. Shin, J. High Energy Phys. 0805100 (2008) [arXiv:hep-ph/0803.2932]

[7] M. Dvornikov and A. Studenikin, Phys. Rev. D 69, 073001 (2004); L.G. Cabral-Rosettic, J. Bernabeu, J. Vidal and A. Zepeda, Eur. Phys. J. C 12, 633 (2000); A. Denner, G. Weiglein and S. Dittmaier, Nucl. Phys. B440, 95 (1995)

[8] E.W. Kolb and M.S. Turner, The Early Universe (Addison-Wesley Redwood City, 1989)

[9] D.N. Spergel et al., (WMAP Collaboration), Astrophys. J Suppl. 170, 377 (2007) arXiv:astro-ph/0603449

[10] P. Gondolo and G. Gelmini, Nucl. Phys. B360, 145 (1991)

[11] J.R. Ellis, A. Ferstl and K.A. Olive, Phys. Lett. B 481, 304 (2000) arXiv:hep-ph/0001005

[12] K. Freese, J. Frieman and A. Gould, Phys. Rev. D 37, 3388 (1988)

[13] K. Griest, Phys. Rev. D 37, 2703 (1988); K. Freese, J.A. Frieman, and A. Gould, Phys. Rev. D 37, 3388 (1988) ; A. Drukier, K. Freese and D. Spergel, Phys. Rev. D 33, 3495 (1986)

[14] J. Angle et al. (XENON10 Collaboration), Phys. Rev. Lett. 101, 091301 (2008) [arXiv:astro$\mathrm{ph} / 0802.3530]$

[15] Z. Ahmed et al. (CDMS Collaboration), Phys. Rev. Lett. 102, 011301 (2009) [arXiv:astroph/0802.3530] ; Phys. Rev. D 73, 011102 (2006) arXiv:astro-ph/0509269 
[16] D.S. Akerib et al. (CDMS Collaboration), Nucl. Instrum. Meth. A 55, 411 (2006) arXiv:astro-ph/0503583

[17] V.I. Dimitrov, J. Engel and S. Pittel, Phys. Rev. D 51, R291 (1995) ; J. Engel, Phys. Lett. B264, 114 (1991) ; M.T. Ressel et al., Phys. Rev. D 48, 5519 (1993)

[18] M.T. Ressell and D.J. Dean, Phy. Rev. C 56, 535 (1997) [arXiv:hep-ph/970229] ; M.A. Nikolaev and H.V. Klapdor-Kleingrothaus, Z. Phys. A 345, 183 (1993)

[19] proceeding 